Journal of Algebra Combinatorics Discrete Structures and Applications

\title{
On the spectral characterization of kite graphs*
}

\section{Research Article}

\author{
Sezer Sorgun, Hatice Topcu
}

\begin{abstract}
The Kite graph, denoted by Kite $_{p, q}$ is obtained by appending a complete graph $K_{p}$ to a pendant vertex of a path $P_{q}$. In this paper, firstly we show that no two non-isomorphic kite graphs are cospectral w.r.t the adjacency matrix. Let $G$ be a graph which is cospectral with Kite $_{p, q}$ and let $w(G)$ be the clique number of $G$. Then, it is shown that $w(G) \geq p-2 q+1$. Also, we prove that Kite $_{p, 2}$ graphs are determined by their adjacency spectrum.
\end{abstract}

2010 MSC: $05 \mathrm{C} 50,05 \mathrm{C} 75$

Keywords: Kite graph, Cospectral graphs, Clique number, Determined by adjacency spectrum

\section{Introduction}

All of the graphs considered here are simple and undirected. Let $G=(V, E)$ be a graph with vertex set $V(G)=\left\{v_{1}, v_{2}, \ldots, v_{n}\right\}$ and edge set $E(G)$. For a given graph $F$, if $G$ does not contain $F$ as an induced subgraph, then $G$ is called $F$ - free. A complete subgraph of $G$ is a clique of $G$. The clique number of $G$ is the number of the vertices in the largest clique of $G$ and it is denoted by $w(G)$. Let $A(G)$ be the (0,1)-adjacency matrix of $G$ and $d_{k}$ denotes the degree of the vertex $v_{k}$. The polynomial $P_{A(G)}(\lambda)=\operatorname{det}(\lambda I-A(G))$ is the adjacency characteristic polynomial of $G$, where $I$ is the identity matrix. Eigenvalues of the matrix $A(G)$ are adjacency eigenvalues. Since $A(G)$ is real and symmetric matrix, adjacency eigenvalues are all real numbers and could be ordered as $\lambda_{1}(A(G)) \geq \lambda_{2}(A(G)) \geq$ $\ldots \geq \lambda_{n}(A(G))$. Adjacency spectrum of the graph $G$ consists of the adjacency eigenvalues with their multiplicities. The largest absolute value of the adjacency eigenvalues of a graph is known as its adjacency spectral radius. Two graphs $G$ and $H$ are said to be cospectral if they have same spectrum (i.e., same characteristic polynomial). A graph $G$ is determined by its adjacency spectrum, shortly $D A S$, if every graph cospectral with $G$ w.r.t the adjacency matrix, is isomorphic to $G$. It is conjectured in [5] that almost all graphs are determined by their spectrum, $D S$ for short. But it is difficult to show that a given

* This work was supported by the Nevsehir Haci Bektas Veli Univesity Coordinatorship of Scientific Research Projects (No. NEULUP15F17).

Sezer Sorgun (Corresponding Author), Hatice Topcu; Department of Mathematics, Nevşehir Hacı Bektaş Veli University, Nevşehir 50300, Turkey (email: srgnrzs@gmail.com, haticekamittopcu@gmail.com). 
graph is $D S$. Up to now, some graphs are proved to be $D S[1,2,4-11,13,15]$. Recently, some papers have appeared in the literature that researchers focus on some special graphs (oftenly under some conditions) and prove that these special graphs are $D S$ or non-DS $[1,2,6-11,13,15]$. For a recent survey, one can see $[5]$.

The Kite graph, denoted by Kite $_{p, q}$, is obtained by appending a complete graph with $p$ vertices $K_{p}$ to a pendant vertex of a path graph with $q$ vertices $P_{q}$. If $q=1$, it is called short kite graph.

In this paper, firstly we obtain the characteristic polynomial of kite graphs and show that no two non-isomorphic kite graphs are cospectral w.r.t the adjacency matrix. Then for a given graph $G$ which is cospectral with Kite $_{p, q}$, the clique number of $G$ is $w(G) \geq p-2 q+1$. Also we prove that Kite $e_{p, 2}$ graphs are $D A S$ for all $p$.

\section{Preliminaries}

First, we give some lemmas that will be used in the next sections of this paper.

Lemma 2.1. [8] Let $x_{1}$ be a pendant vertex of a graph $G$ and $x_{2}$ be the vertex which is adjacent to $x_{1}$. Let $G_{1}$ be the induced subgraph obtained from $G$ by deleting the vertex $x_{1}$. If $x_{1}$ and $x_{2}$ are deleted, the induced subgraph $G_{2}$ is obtained. Then,

$$
P_{A(G)}(\lambda)=\lambda P_{A\left(G_{1}\right)}(\lambda)-P_{A\left(G_{2}\right)}(\lambda)
$$

Lemma 2.2. [4] For nxn matrices $A$ and $B$, followings are equivalent:

(i) $A$ and $B$ are cospectral

(ii) $A$ and $B$ have the same characteristic polynomial

(iii) $\operatorname{tr}\left(A^{i}\right)=\operatorname{tr}\left(B^{i}\right)$ for $i=1,2, \ldots, n$

Lemma 2.3. [4] For the adjacency matrix of a graph $G$, following parameters can be deduced from the spectrum;

(i) the number of vertices

(ii) the number of edges

(iii) the number of closed walks of any fixed length.

Theorem 2.4. [14] If a given connected graph $G$ has the same order, same clique number and same spectral radius with Kite $_{p, q}$ then $G$ is isomorphic to Kite in $_{\text {. }}$.

In the rest of the paper, we denote the number of subgraphs of a graph $G$ which are isomorphic to complete graph $K_{3}$ by $t(G)$.

Theorem 2.5. [14] For any integers $p \geq 3$ and $q \geq 1$, if we denote the spectral radius of $A\left(K\right.$ ite $\left.e_{p, q}\right)$ with $\rho\left(\right.$ Kite $\left._{p, q}\right)$ then

$$
p-1+\frac{1}{p^{2}}+\frac{1}{p^{3}}<\rho\left(\text { Kite }_{p, q}\right)<p-1+\frac{1}{4 p}+\frac{1}{p^{2}-2 p}
$$

Theorem 2.6. [12] Let $G$ be a graph with $n$ vertices, $m$ edges and spectral radius $\mu$. If $G$ is $K_{r+1}-f r e e$, then

$$
\mu \leq \sqrt{2 m\left(\frac{r-1}{r}\right)}
$$

Lemma 2.7. [3](Interlacing Lemma) If $G$ is a graph on $n$ vertices with eigenvalues $\lambda_{1}(G) \geq \ldots \geq$ $\lambda_{n}(G)$ and $H$ is an induced subgraph on $m$ vertices with eigenvalues $\lambda_{1}(H) \geq \ldots \geq \lambda_{m}(H)$, then for $i=1, \ldots, m$

$$
\lambda_{i}(G) \geq \lambda_{i}(H) \geq \lambda_{n-m+i}(G)
$$


Lemma 2.8. [3] A connected graph with the largest adjacency eigenvalue less than 2 are precisely induced subgraphs of the Smith graphs shown in Figure 1.

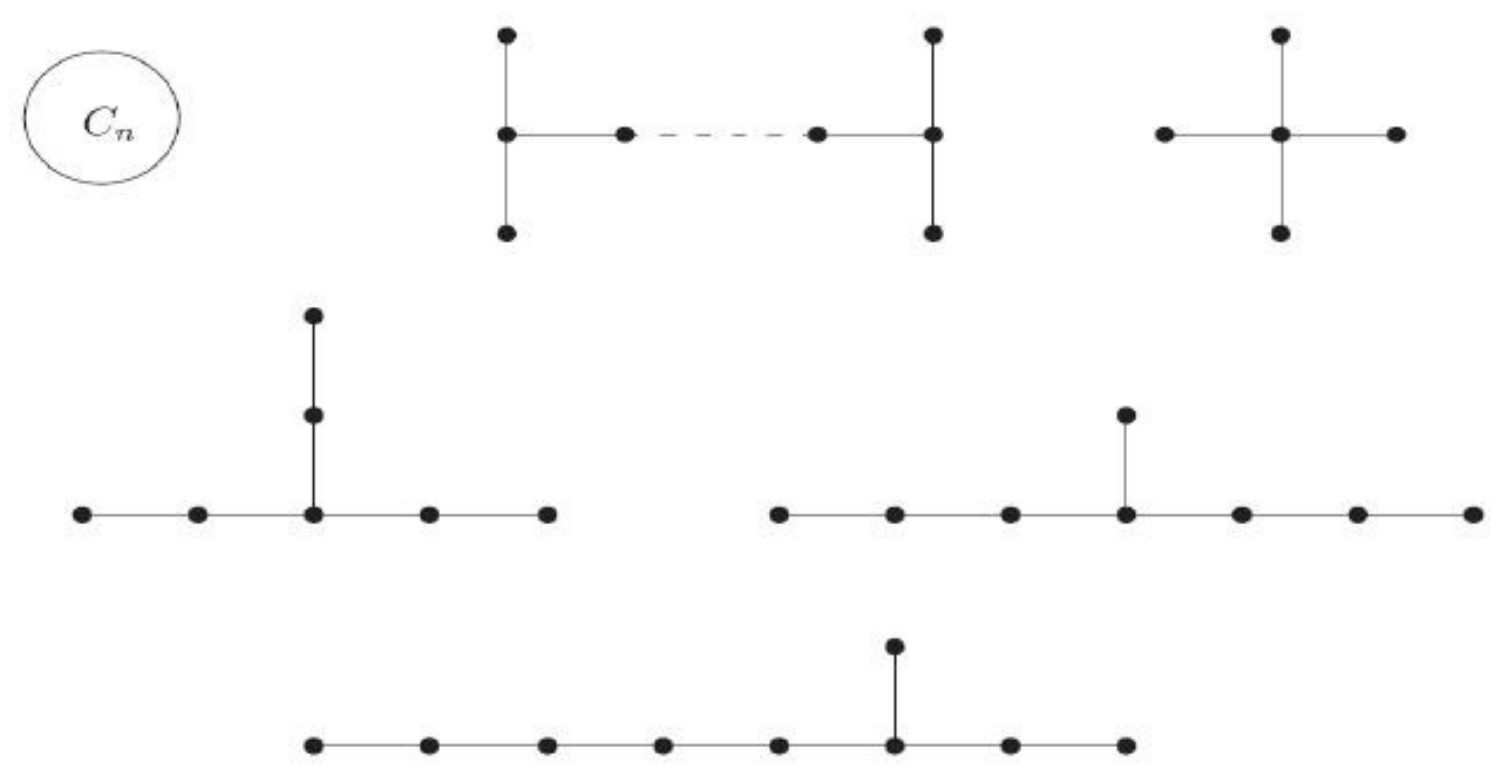

Figure 1. Smith graphs

\section{Characteristic polynomial of kite graphs}

We use the method similar to that given in [8] to obtain the general form of characteristic polynomials of Kite $_{p, q}$ graphs. Obviously, if we delete the vertex with one degree from short kite graph, the induced subgraph will be the complete graph $K_{p}$. Then, by deleting the vertex with one degree and its adjacent vertex, we obtain the complete graph $K_{p-1}$ with $p-1$ vertices. From Lemma 2.1, we get

$$
\begin{aligned}
P_{A\left(\text { Kite }_{p, 1}\right)}(\lambda) & =\lambda P_{A\left(K_{p}\right)}(\lambda)-P_{A\left(K_{p-1}\right)}(\lambda) \\
& =\lambda(\lambda-p+1)(\lambda+1)^{p-1}-\left[(\lambda-p+2)(\lambda+1)^{p-2}\right] \\
& =(\lambda+1)^{p-2}\left[\left(\lambda^{2}-\lambda p+\lambda\right)(\lambda+1)-\lambda+p-2\right] \\
& =(\lambda+1)^{p-2}\left[\lambda^{3}-(p-2) \lambda^{2}-\lambda p+p-2\right] .
\end{aligned}
$$

Similarly for Kite $_{p, 2}$, induced subgraphs will be Kite $_{p, 1}$ and $K_{p}$ respectively. By Lemma 2.1, we get

$$
\begin{aligned}
P_{A\left(\text { Kite }_{p, 2}\right)}(\lambda) & \left.=\lambda P_{A\left(\text { Kite }_{p, 1}\right)}(\lambda)-P_{A\left(K_{p}\right)}\right)(\lambda) \\
& \left.=\lambda\left(\lambda P_{A\left(K_{p}\right)}(\lambda)-P_{A\left(K_{p-1}\right)}(\lambda)\right)-P_{A\left(K_{p}\right)}\right)(\lambda) \\
& =\left(\lambda^{2}-1\right) P_{A\left(K_{p}\right)}(\lambda)-\lambda P_{A\left(K_{p-1}\right)}(\lambda) .
\end{aligned}
$$

By using these polynomials, we calculate the characteristic polynomial of Kite $_{p, q}$ where $n=p+q$. Again, by Lemma 2.1 we have

$$
P_{A\left(\text { Kite }_{p, 1}\right)}(\lambda)=\lambda P_{A\left(K_{p}\right)}(\lambda)-P_{A\left(K_{p-1}\right)}(\lambda)
$$


Coefficients of above equation are $b_{1}=-1, a_{1}=\lambda$. Simultaneously, we get

$$
P_{A\left(K_{i t e}, 2\right)}(\lambda)=\left(\lambda^{2}-1\right) P_{A\left(K_{p}\right)}(\lambda)-\lambda P_{A\left(K_{p-1}\right)}(\lambda)
$$

and coefficients of above equation are $b_{2}=-a_{1}=-\lambda, a_{2}=\lambda a_{1}-1=\lambda^{2}-1$. Then for Kite $e_{p, 3}$, we have

$$
\begin{aligned}
P_{A\left(\text { Kite }_{p, 3}\right)}(\lambda) & \left.=\lambda P_{A\left(\text { Kite }_{p, 2}\right)}(\lambda)-P_{A\left(\text { Kite }_{p, 1}\right)}\right)(\lambda) \\
& =\left(\lambda\left(\lambda^{2}-1\right)-\lambda\right) P_{A\left(K_{p}\right)}(\lambda)-\left(\left(\lambda^{2}-1\right) P_{A\left(K_{p-1}\right)}(\lambda)\right)
\end{aligned}
$$

and coefficients of above equation are $b_{3}=-a_{2}=-\left(\lambda^{2}-1\right), a_{3}=\lambda a_{2}-a_{1}=\lambda\left(\lambda^{2}-1\right)-\lambda$. In the following steps, for $n \geq 3, a_{n}=\lambda a_{n-1}-a_{n-2}$. From this difference equation, we get

$$
a_{n}=\sum_{k=0}^{n}\left(\frac{\lambda+\sqrt{\lambda^{2}-4}}{2}\right)^{k}\left(\frac{\lambda-\sqrt{\lambda^{2}-4}}{2}\right)^{n-k}
$$

Now, let $\lambda=2 \cos \theta$ and $u=e^{i \theta}$. Then, we have

$$
a_{n}=\sum_{k=0}^{n} u^{2 k-n}=\frac{u^{-n}\left(1-u^{2 n+2}\right)}{1-u^{2}}
$$

and by calculation the characteristic polynomial of any kite graph Kite $_{p, q}$ where $n=p+q$ is

$$
\begin{aligned}
P_{A\left(\text { Kite }_{p, q}\right)}\left(u+u^{-1}\right)= & a_{n-p} P_{A\left(K_{p}\right)}\left(u+u^{-1}\right)-a_{n-p-1} P_{A\left(K_{p-1}\right)}\left(u+u^{-1}\right) \\
= & \frac{u^{-n+p}\left(1-u^{2 n-2 p+2}\right)}{1-u^{2}} \cdot\left(\left(u+u^{-1}-p+1\right) \cdot\left(u+u^{-1}+1\right)^{p-1}\right) \\
& -\frac{u^{-n+p+1}\left(1-u^{2 n-2 p+4}\right)}{1-u^{2}} \cdot\left(\left(u+u^{-1}-p+2\right) \cdot\left(u+u^{-1}+1\right)^{p-2}\right) \\
= & \frac{u^{-n+p}\left(1+u-u^{-1}\right)^{p-2}}{1-u^{2}}\left[(2-p) \cdot\left(1+u^{-1}-u^{2 n-2 p+2}-u^{2 n-2 p+3}\right)\right. \\
& \left.+\left(u^{-2}-u^{2 n-2 p+4}\right)\right] \\
= & \frac{u^{-q}\left(1+u-u^{-1}\right)^{p-2}}{1-u^{2}}\left[(2-p) \cdot\left(1+u^{-1}-u^{2 q+2}-u^{2 q+3}\right)\right. \\
& \left.+\left(u^{-2}-u^{2 q+4}\right)\right] .
\end{aligned}
$$

Theorem 3.1. No two non-isomorphic kite graphs have the same adjacency spectrum.

Proof. Assume that there are two cospectral kite graphs with number of vertices respectively, $p_{1}+q_{1}$ and $p_{2}+q_{2}$. Since they are cospectral, they must have same number of vertices and same characteristic polynomials. Hence, $n=p_{1}+q_{1}=p_{2}+q_{2}$ and we get

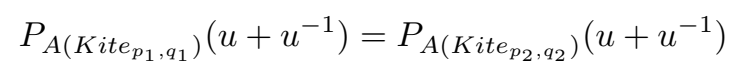

i.e.,

$$
\begin{aligned}
& \frac{u^{-n+p_{1}}\left(1+u-u^{-1}\right)^{p_{1}-2}}{1-u^{2}}\left[\left(2-p_{1}\right) \cdot\left(1+u^{-1}-u^{2 n-2 p_{1}+2}-u^{2 n-2 p_{1}+3}\right)\right. \\
& \left.+\left(u^{-2}-u^{2 n-2 p_{1}+4}\right)\right] \\
= & \frac{u^{-n+p_{2}}\left(1+u-u^{-1}\right)^{p_{2}-2}}{1-u^{2}}\left[\left(2-p_{2}\right) \cdot\left(1+u^{-1}-u^{2 n-2 p_{2}+2}-u^{2 n-2 p_{2}+3}\right)\right. \\
& \left.+\left(u^{-2}-u^{2 n-2 p_{2}+4}\right]\right)
\end{aligned}
$$


i.e.,

$$
\begin{aligned}
& u^{p_{1}} \cdot\left(1+u-u^{-1}\right)^{p_{1}} \cdot\left[\left(2-p_{1}\right) \cdot\left(1+u^{-1}-u^{2 n-2 p_{1}+2}-u^{2 n-2 p_{1}+3}\right)\right. \\
& \left.+\left(u^{-2}-u^{2 n-2 p_{1}+4}\right)\right] \\
= & u^{p_{2}} \cdot\left(1+u-u^{-1}\right)^{p_{2}} \cdot\left[\left(2-p_{2}\right) \cdot\left(1+u^{-1}-u^{2 n-2 p_{2}+2}-u^{2 n-2 p_{2}+3}\right)\right. \\
& \left.+\left(u^{-2}-u^{2 n-2 p_{2}+4}\right)\right]
\end{aligned}
$$

Let $p_{1}>p_{2}$. It follows that $n-p_{2}>n-p_{1}$. Then, we have

$$
\begin{aligned}
& u^{p_{1}-p_{2}} \cdot\left(1+u-u^{-1}\right)^{p_{1}-p_{2}}\left\{\left[\left(2-p_{1}\right) \cdot\left(1+u^{-1}-u^{2 n-2 p_{1}+2}-u^{2 n-2 p_{1}+3}\right)\right.\right. \\
& \left.+\left(u^{-2}-u^{2 n-2 p_{1}+4}\right)\right]-\left[\left(2-p_{2}\right) \cdot\left(1+u^{-1}-u^{2 n-2 p_{2}+2}-u^{2 n-2 p_{2}+3}\right)\right. \\
& \left.\left.+\left(u^{-2}-u^{2 n-2 p_{2}+4}\right)\right]\right\}=0
\end{aligned}
$$

By using the fact that $u \neq 0$ and $1+u+u^{-1} \neq 0$, we get

$$
\begin{aligned}
f(u)= & {\left[\left(2-p_{1}\right) \cdot\left(1+u^{-1}-u^{2 n-2 p_{1}+2}-u^{2 n-2 p_{1}+3}\right)+\left(u^{-2}-u^{2 n-2 p_{1}+4}\right)\right] } \\
& -\left[\left(2-p_{2}\right) \cdot\left(1+u^{-1}-u^{2 n-2 p_{2}+2}-u^{2 n-2 p_{2}+3}\right)+\left(u^{-2}-u^{2 n-2 p_{2}+4}\right)\right] \\
= & 0
\end{aligned}
$$

Since $f(u)=0$, the derivation of $\left(2 n-2 p_{2}+5\right)$ th of $f$ equals to zero again. Thus, we have

$$
\left[\left(p_{1}-2\right)\left(2 n-2 p_{2}+4\right) !\left(u^{-2 n+2 p_{2}-6}\right)\right]-\left[\left(p_{2}-2\right) \cdot\left(2 n-2 p_{2}+4\right) !\left(u^{-2 n+2 p_{2}-6}\right)\right]=0
$$

i.e.,

$$
\left[\left(p_{1}-2\right)-\left(p_{2}-2\right)\right] \cdot\left(u^{-2 n+2 p_{2}-6}\right)=0
$$

i.e.,

$$
p_{1}=p_{2}
$$

since $u \neq 0$. This is a contradiction with our assumption that is $p_{1}>p_{2}$. For $p_{2}>p_{1}$, we get the similar contradiction. So $p_{1}$ must be equal to $p_{2}$. Hence $q_{1}=q_{2}$ and these graphs are isomorphic.

\section{Spectral characterization of $\mathrm{Kite}_{p, 2}$ graphs}

Lemma 4.1. Let $G$ be a graph which is cospectral with Kite $_{p, q}$. Then we get

$$
w(G) \geq p-2 q+1
$$

Proof. Since $G$ is cospectral with Kite $_{p, q}$, from Lemma 2.3, $G$ has the same number of vertices, same number of edges and same spectrum with Kite $p, q$. So, if $G$ has $n$ vertices and $m$ edges, $n=p+q$ and $m=\left(\begin{array}{l}p \\ 2\end{array}\right)+q=\frac{p^{2}-p+2 q}{2}$. Also, $\rho(G)=\rho\left(\right.$ Kite $\left._{p, q}\right)$. From Theorem 2.6, we say that if $\mu>\sqrt{2 m\left(\frac{r-1}{r}\right)}$ then $G$ isn't $K_{r+1}-$ free. It means that, $G$ contains $K_{r+1}$ as an induced subgraph. Now, we claim that for $r<p-2 q, \sqrt{2 m\left(\frac{r-1}{r}\right)}<\rho(G)$. By Theorem 2.5, we've already known that $p-1+\frac{1}{p^{2}}+\frac{1}{p^{3}}<\rho(G)$. Hence, we need to show that $\sqrt{2 m\left(\frac{r-1}{r}\right)}<p-1+\frac{1}{p^{2}}+\frac{1}{p^{3}}$, when $r<p-2 q$. Indeed, 


$$
\begin{aligned}
\left(\sqrt{\left.2 m\left(\frac{r-1}{r}\right)\right)^{2}-\left(p-1+\frac{1}{p^{2}}+\frac{1}{p^{3}}\right)^{2}=}\right. & \left(p^{2}-p+2 q\right)(r-1)-r\left(p-1+\frac{1}{p^{2}}+\frac{1}{p^{3}}\right)^{2} \\
= & \left(p^{2}-p+2 q\right)(r-1)- \\
& \left(\frac{r\left(p^{2}+p^{3}\right)}{p^{5}}\right)\left(2(p-1)+\frac{\left(p^{2}+p^{3}\right)}{p^{5}}\right) \\
= & \left(p r-p^{2}+p+(2 q-1) r-2 q\right)- \\
& \left(\frac{r\left(p^{2}+p^{3}\right)}{p^{5}}\right)\left(2(p-1)+\frac{\left(p^{2}+p^{3}\right)}{p^{5}}\right)
\end{aligned}
$$

By the help of Mathematica, for $r<p-2 q$ we can see

$$
\left(p r-p^{2}+p+(2 q-1) r-2 q\right)-\left(\frac{r\left(p^{2}+p^{3}\right)}{p^{5}}\right)\left(2(p-1)+\frac{\left(p^{2}+p^{3}\right)}{p^{5}}\right)<0
$$

i.e.,

$$
\left(\sqrt{2 m\left(\frac{r-1}{r}\right)}\right)^{2}-\left(p-1+\frac{1}{p^{2}}+\frac{1}{p^{3}}\right)^{2}<0
$$

i.e.,

$$
\left(\sqrt{2 m\left(\frac{r-1}{r}\right)}\right)^{2}<\left(p-1+\frac{1}{p^{2}}+\frac{1}{p^{3}}\right)^{2}
$$

Since $\sqrt{2 m\left(\frac{r-1}{r}\right)}>0$ and $p-1+\frac{1}{p^{2}}+\frac{1}{p^{3}}>0$, we get

$$
\sqrt{2 m\left(\frac{r-1}{r}\right)}<p-1+\frac{1}{p^{2}}+\frac{1}{p^{3}}<\rho(G)
$$

Thus, we proved our claim and so $G$ contains $K_{r+1}$ as an induced subgraph such that $r<p-2 q$. Consequently, $w(G) \geq p-2 q+1$.

Theorem 4.2. Kite $_{p, 2}$ graphs are determined by their adjacency spectrum for all $p$.

Proof. If $p=1$ or $p=2$, Kite $e_{p, 2}$ graphs are actually the path graphs $P_{3}$ or $P_{4}$. Also if $p=3$, then we obtain the lollipop graph $H_{5,3}$. As is known, these graphs are already $D A S$ [8]. Hence we will continue our proof for $p \geq 4$. Adjacency characteristic polynomial of $K_{i t e_{p, 2}}$ is as below,

$$
P_{A\left(\text { Kite }_{p, 2}\right)}(\lambda)=(\lambda+1)^{p-2}\left[\lambda^{4}+(2-p) \lambda^{3}-(p+1) \lambda^{2}+(2 p-4) \lambda+p-1\right]
$$

By calculation, for the adjacency eigenvalues of Kite $_{p, 2}$, we obtain the following facts; $p-1<\lambda_{1}\left(A\left(\right.\right.$ Kite $\left.\left._{p, 2}\right)\right)<p, 0<\lambda_{2}\left(\right.$ A $\left.\left.\left._{\text {Kite }}\right), 2\right)\right)<2, \lambda_{3}\left(\right.$ A $\left.\left._{\text {Kite }}, 2\right)\right)<0, \lambda_{4}\left(A\left(\right.\right.$ Kite $\left.\left._{p, 2}\right)\right)=\ldots=$ $\lambda_{p+1}\left(A\left(\right.\right.$ Kite $\left.\left._{p, 2}\right)\right)=-1$ and $\lambda_{p-1}\left(A\left(\right.\right.$ Kite $\left.\left._{p, 2}\right)\right)<-1$.

For a given graph $G$ with $n$ vertices and $m$ edges, assume that $G$ is cospectral with Kite $_{p, 2}$. Then by Lemma $2.3, n=p+2, m=\left(\begin{array}{c}p \\ 2\end{array}\right)+2=\frac{p^{2}-p+4}{2}$ and $t(G)=t\left(\right.$ Kite $\left._{p, 2}\right)=\left(\begin{array}{c}p \\ 3\end{array}\right)=\frac{p^{3}-3 p^{2}+2 p}{6}$. From 
Lemma 4.1, $w(G) \geq p-2 q+1$. When $q=2, w(G) \geq p-3=n-5$. It's well-known that complete graph $K_{n}$ is $D S$. So $w(G) \neq n$. If $w(G)=n-1=p+1$, then $G$ contains at least one clique with size $p-1$. It means that the edge number of $G$ is greater than or equal to $\left(\begin{array}{c}p+1 \\ 2\end{array}\right)$. But it is a contradiction since $\left(\begin{array}{c}p+1 \\ 2\end{array}\right)>\left(\begin{array}{c}p \\ 2\end{array}\right)+2=m$. Hence, $w(G) \neq n-1$. Because of these facts, we get $p-3 \leq w(G) \leq p$. From interlacing lemma, $G$ can not contain the graphs in the following figure as an induced subgraph because $\lambda_{3}\left(G_{1}\right)=\lambda_{3}\left(G_{2}\right)=0$.
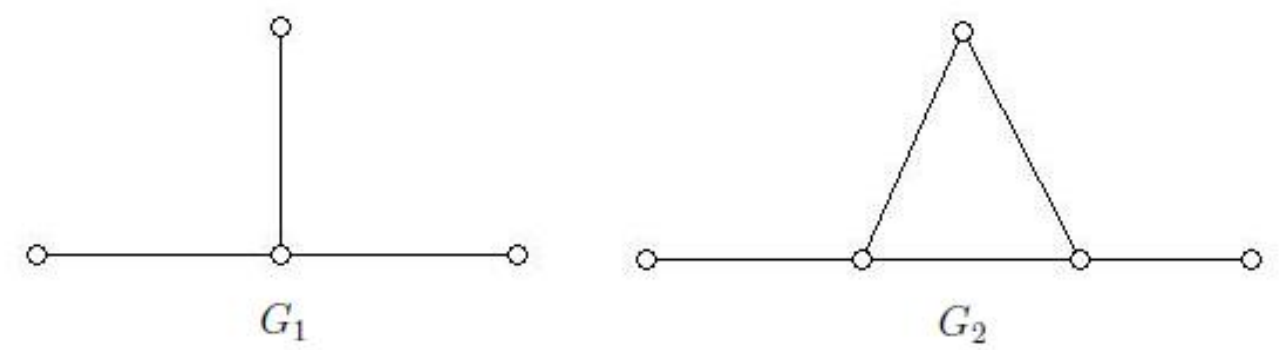

\section{Figure 2. Graphs $\mathbf{G}_{1}$ and $\mathrm{G}_{2}$}

If $G$ is disconnected, from Lemma 2.8, components of $G$ except one of them must be induced subgraphs of Smith graphs. Clearly, this is impossible because $G_{1}$ is forbidden and any path graph (since they have symmetric eigenvalues) can not be a component of $G$. Hence $G$ must be a connected graph. If $w(G)=p$, then by Theorem 2.4., $G \cong K i t e_{p, 2}$. So we continue for $w(G)<p$. Since $w(G) \geq p-3$, $G$ contains at least one clique with size at least $p-3$. This clique is denoted by $K_{w(G)}$. There may be at most five vertices out of the clique $K_{w(G)}$. Let us label these five vertices respectively with $1,2,3,4,5$ and call the set of these five vertices with $A$. So, we get $|A| \leq 5$. Moreover, $\forall i, j \in A$ we get $i \sim j$ since $G_{1}, G_{2}$ are not induced subgraphs of $G$ and there is no isolated vertex in $G$. Then, we can say that $p \geq 6$ since $w(G) \geq p-3$.

For $i \in A, x_{i}$ denotes the number of adjacent vertices of $i$ in $K_{w(G)}$. By the fact that $p-1 \geq w(G) \geq$ $p-3$, for all $i \in A$ we say

$$
x_{i} \leq w(G)-|A|+1
$$

Also, $x_{i \wedge j}$ denotes the number of common adjacent vertices in $K_{w(G)}$ of $i$ and $j$ such that $i, j \in A$ and $i<j$. Similarly, if $i \sim j$ then

$$
x_{i \wedge j} \leq w(G)-|A|
$$

Let $d$ denotes the number of edges between the vertices of $\mathrm{A}$ and $K_{w(G)}$, also $\alpha$ denotes the number of cliques with size 3 which are not contained by $A$ or $K_{w(G)}$. Then, we get

$$
m=\left(\begin{array}{c}
p \\
2
\end{array}\right)+2=\left(\begin{array}{c}
w(G) \\
2
\end{array}\right)+\left(\begin{array}{c}
|A| \\
2
\end{array}\right)+d
$$

Similarly, we get

$$
t(G)=\left(\begin{array}{c}
p \\
3
\end{array}\right)=\left(\begin{array}{c}
w(G) \\
3
\end{array}\right)+\left(\begin{array}{c}
|A| \\
3
\end{array}\right)+\alpha .
$$

On the other hand for $\alpha$ and $d$, we have

$$
d=\sum_{i=1}^{|A|} x_{i}
$$


and

$$
\alpha=\sum_{i=1}^{|A|}\left(\begin{array}{c}
x_{i} \\
2
\end{array}\right)+\sum_{i \sim j} x_{i \wedge j} .
$$

If $w(G)=p-3$ then $|A|=5$ and so $p \geq 8$. Thus we have

$$
d=3 p-14
$$

and

$$
\alpha=\left(\begin{array}{c}
p \\
3
\end{array}\right)-\left(\begin{array}{c}
p-3 \\
3
\end{array}\right)-10=\frac{3 p^{2}}{2}-\frac{15 p}{2} .
$$

From (1),(2),(5),(6) and (7) we have

$$
\begin{aligned}
\alpha=\sum_{i=1}^{5}\left(\begin{array}{c}
x_{i} \\
2
\end{array}\right)+\sum_{i \sim j} x_{i \wedge j} & \leq 3\left(\begin{array}{c}
p-7 \\
2
\end{array}\right)+\left(\begin{array}{l}
7 \\
2
\end{array}\right)+2 \sum_{i=1}^{5} x_{i} \\
& =3\left(\begin{array}{c}
p-7 \\
2
\end{array}\right)+\left(\begin{array}{l}
7 \\
2
\end{array}\right)+6 p-28 \\
& =\frac{3 p^{2}-33 p}{2}+77 .
\end{aligned}
$$

But obviously for $p=8$ this result gives contradiction. Also for $p>8$,

$$
\frac{3 p^{2}-33 p}{2}+77<\frac{3 p^{2}-15 p}{2}=\alpha .
$$

So this is again a contradiction.

$$
\begin{gathered}
\text { If } w(G)=p-2 \text { then }|A|=4 \text { and so } p \geq 7 \text {. Thus we have } \\
\qquad d=2 p-7
\end{gathered}
$$

and

$$
\alpha=\left(\begin{array}{c}
p \\
3
\end{array}\right)-\left(\begin{array}{c}
p-2 \\
3
\end{array}\right)-4=p^{2}-4 p
$$

On the other hand we have

$$
\begin{aligned}
\alpha=\sum_{i=1}^{4}\left(\begin{array}{c}
x_{i} \\
2
\end{array}\right)+\sum_{i \sim j} x_{i \wedge j} & \leq 2\left(\begin{array}{c}
p-5 \\
2
\end{array}\right)+\left(\begin{array}{l}
3 \\
2
\end{array}\right)+2 \sum_{i=1}^{4} x_{i} \\
& =p^{2}-7 p+19 .
\end{aligned}
$$

Clearly for $p \geq 7$,

$$
p^{2}-7 p+19<p^{2}-4 p=\alpha
$$

So this is a contradiction.

Similarly, if $w(G)=p-1$ then $|A|=3$ and so $p \geq 6$. Hence we have

$$
d=p-2
$$

and

$$
\alpha=\frac{p^{2}-3 p}{2} .
$$


Also we have

$$
\begin{aligned}
\alpha=\sum_{i=1}^{3}\left(\begin{array}{c}
x_{i} \\
2
\end{array}\right)+\sum_{i \sim j} x_{i \wedge j} & \leq\left(\begin{array}{c}
p-3 \\
2
\end{array}\right)+p-2 \\
& =\frac{p^{2}-5 p}{2}+4 .
\end{aligned}
$$

Clearly for $p \geq 6$,

$$
\frac{p^{2}-5 p}{2}+4<\frac{p^{2}-3 p}{2}=\alpha
$$

Again we obtain a contradiction.

By all of these facts, we can conclude that our assumption is actually false, then $w(G) \nless p$. Hence $w(G)=p$ and so that by Theorem 2.4., $G \cong$ Kite $_{p, 2}$.

In the final of the paper, we give a conjecture below.

Conjecture 4.3. For $q>2$, Kite $e_{p, q}$ graphs are DAS.

Acknowledgment: The authors are grateful to the referees for many suggestions which led to an improved version of this paper.

\section{References}

[1] R. Boulet, B. Jouve, The lollipop graph is determined by its spectrum, Electron. J. Combin. 15(1) (2008) Research Paper 74, 43 pp.

[2] M. Camara, W. H. Haemers, Spectral characterizations of almost complete graphs, Discrete Appl. Math. 176 (2014) 19-23.

[3] M. D. Cvetkovic, P. Rowlinson, S. Simic, An Introduction to the Theory of Graph Spectra, Cambridge University Press, 2010.

[4] E.R. van Dam, W. H. Haemers, Which graphs are determined by their spectrum?, Linear Algebra Appl. 373 (2003) 241-272.

[5] E.R. van Dam, W. H. Haemers, Developments on spectral characterizations of graphs, Discrete Math. 309(3) (2009) 576-586.

[6] M. Doob, W. H. Haemers, The complement of the path is determined by its spectrum, Linear Algebra Appl. 356(1-3) (2002) 57-65.

[7] N. Ghareghani, G. R. Omidi, B. Tayfeh-Rezaie, Spectral characterization of graphs with index at most $\sqrt{2+\sqrt{5}}$, Linear Algebra Appl. 420(2-3) (2007) 483-486.

[8] W. H. Haemers, X. Liu, Y. Zhang, Spectral characterizations of lollipop graphs, Linear Algebra Appl. 428(11-12) (2008) 2415-2423.

[9] F. Liu, Q. Huang, J. Wang, Q. Liu, The spectral characterization of $\infty$-graphs, Linear Algebra Appl. 437(7) (2012) 1482-1502.

[10] M. Liu, H. Shan, K. Ch. Das, Some graphs determined by their (signless) Laplacian spectra, Linear Algebra Appl. 449 (2014) 154-165.

[11] X. Liu, Y. Zhang, X. Gui, The multi-fan graphs are determined by their Laplacian spectra, Discrete Math. 308(18) (2008) 4267-4271.

[12] V. Nikiforov, Some inequalities for the largest eigenvalue of a graph, Combin. Probab. Comput. 11(2) (2002) 179-189.

[13] G. R. Omidi, On a signless Laplacian spectral characterization of T-shape trees, Linear Algebra Appl. 431(9) (2009) 1607-1615. 
[14] D. Stevanovic, P. Hansen, The minimum spectral radius of graphs with a given clique number, Electron. J. Linear Algebra. 17 (2008) 110-117.

[15] X. Zhang, H. Zhang, Some graphs determined by their spectra, Linear Algebra Appl. 431(9) (2009) $1443-1454$. 